\title{
Akap12beta supports asymmetric heart development via modulating the Kupffer's vesicle formation in zebrafish
}

\author{
Jeong-gyun Kim ${ }^{1}$, Hyun-Ho Kim ${ }^{1,2}$ E Sung-Jin Bae , $^{1, *}$ \\ ${ }^{1}$ College of Pharmacy and Research Institute of Pharmaceutical Sciences, Seoul National University, Seoul 08826, ${ }^{2}$ Biological and Medical \\ Device Evaluation Team, Korea Testing \& Research Institute, Gwacheon 13810, ${ }^{3}$ Korean Medicine Research Center for Healthy Aging, \\ Pusan National Univerity, Yangsan 50612, Korea
}

The vertebrate body plan is accomplished by left-right asymmetric organ development and the heart is a representative asymmetric internal organ which jogs to the left-side. Kupffer's vesicle (KV) is a spherical left-right organizer during zebrafish embryogenesis and is derived from a cluster of dorsal forerunner cells (DFCs). Cadherin1 is required for collective migration of a DFC cluster and failure of DFC collective migration by Cadherin1 decrement causes KV malformation which results in defective heart laterality. Recently, loss of function mutation of A-kinase anchoring protein 12 (AKAP12) is reported as a high-risk gene in congenital heart disease patients. In this study, we demonstrated the role of akap12 $\beta$ in asymmetric heart development. The akap12 $\beta$, one of the akap12 isoforms, was expressed in DFCs which give rise to KV and akap12 $\beta$ deficient zebrafish embryos showed defective heart laterality due to the fragmentation of DFC clusters which resulted in KV malformation. DFC-specific loss of akap12 $\beta$ also led to defective heart laterality as a consequence of the failure of collective migration by cadherin1 reduction. Exogenous akap $12 \beta$ mRNA not only restored the defective heart laterality but also increased cadherin 1 expression in akap12 $\beta$ morphant zebrafish embryos. Taken together, these findings provide the first experimental evidence that akap12 $\beta$ regulates heart laterality via cadherin1. [BMB Reports 2019; 52(8): 526-531]

\section{INTRODUCTION}

Kupffer's vesicle (KV) is a spherical left-right organizer which appears transiently during an embryonic stage in zebrafish (1). KV originates from a cluster of dorsal forerunner cells (DFCs). Cadherin1 (Cdh1)-mediated adherens junctions sustain cell

${ }^{*}$ Corresponding author. Tel: +82-51-510-8434; Fax: +82-51-5108437; E-mail: Dr.NowOrNever@pusan.ac.kr

https://doi.org/10.5483/BMBRep.2019.52.8.111

Received 15 April 2019, Revised 10 May 2019, Accepted 3 June 2019

Keywords: AKAP12, Asymmetric development, Heart laterality, Kupffer's vesicle (KV), Zebrafish cluster formation between the adjacent DFCs and a cluster of DFCs actively migrates towards the vegetal pole to form KV (2). Then, migrated DFCs attach to the overlying surface epithelium and become polarized to construct a rosette-like structure which contains the lumen at the apical point (3). Finally, cilia-formed and fluid-filled KV expands the internal lumen and motile cilia generate fluid flow in a counterclockwise direction to evoke asymmetric signal(s) such as Nodal, Lefty, and Pitx2 (1).

The vertebrate body plan is accomplished by left-right asymmetric organ development. The heart is a representative asymmetric internal organ which jogs to the left-side and proper positioning during embryonic development is crucial for its function (4). Accordingly, about $\sim 1 \%$ of newborn babies suffer from congenital heart disease (CHD), which has high mortality (5). Dextrocardia, a rare condition in which the apex of the heart is located on the right side of the body, comprises a CHD case with heterotaxy which is often accompanied by asymmetric defects such as a left-sided liver and a right-sided stomach (6).

A-kinase anchoring protein 12 (AKAP12) is a member of the AKAP family proteins, which bind to the regulatory subunit of protein kinase $\mathrm{A}$ (PKA) and holoenzyme localizes to specific locations within the cell. Besides PKA, AKAP12 displays diverse docking sites for protein kinase $\mathrm{C}$, calmodulin, cyclins, $\beta$-1,4-galactosyltransferase, protein phosphatases, the nonreceptor tyrosine kinase Src, and $\beta 2$-adrenergic receptor. In addition, AKAP12 consists of three polybasic domains, four nuclear localization signals, and a nuclear exclusion domain. Therefore, AKAP12 plays various roles in many biological processes including cell migration, cell cycle regulation, barriergenesis, tumor progression, and wound healing (7). Our group previously reported that AKAP 12 regulates the blood-brain and blood-retinal barrier $(8,9)$. This barriergenic property of AKAP12 is also applied to the repair of the central nervous system (CNS) after injury. AKAP12 is strongly expressed in the fibrotic scar during the CNS repair process where it mediates barrier functions (10). Moreover, we and other group reported that akap12 is involved in mesodermal cell shape change, muscle progenitor cell migration and regulation of vascular integrity in zebrafish (11-13).

ISSN: 1976-670X (electronic edition)

Copyright (C) 2019 by the The Korean Society for Biochemistry and Molecular Biology

(c) This is an open-access article distributed under the terms of the Creative Commons Attribution Non-Commercial License (http://creativecommons.org/licenses/by-nc/4.0) which permits unrestricted non-commercial use, distribution, and reproduction in any medium, provided the original work is properly cited. 
Previous $A K A P 12$ studies did not focus on the differential role of the two AKAP12 isoforms, AKAP12 $\alpha$ and AKAP12 3 . Although they only have a small difference in the $\mathrm{N}$-terminal region (less than 5\%), each AKAP12 isoform has an independent promoter (14) and shows distinct spatiotemporal mRNA expression during embryogenesis $(12,13)$. Interestingly, a recent study identified $A K A P 12$ as a loss-of-function mutated gene in CHD patients, which has not been reported in previous reports (15). Here, we demonstrate that akap $12 \beta$, not akap $12 \alpha$, is expressed in DFCs and that loss of akap $12 \beta$ leads to defective heart laterality due to decreased $c d h 1$ expression. This report provides the first experimental evidence of a heart laterality regulatory mechanism by akap $12 \beta$, which might also play a role in human heart heterotaxy.

\section{RESULTS}

\section{$A k a p 12 \beta$ is the major isoform of akap12 during KV development in zebrafish}

Akap $12 \alpha$ and akap12 $\beta$ are two known isoforms of akap12 in zebrafish (Fig. 1A). During embryonic development, each isoform is differentially regulated due to a distinct promoter region (Fig. 1A, B). The expression of akap $12 \beta$ was first observed in the sphere stage, and that of akap $12 \alpha$ was initiated in the bud stage, later than akap12 $\beta$. Interestingly, akap $12 \beta$ morpholino $(\mathrm{MO})$ injection significantly reduced the expression of pan-akap12 at $75 \%$ epiboly stage, while akap12 $\alpha \mathrm{MO}$ injection did not affect the pan-akap12 expression (Fig. 1C, D).

Next, we investigated the spatiotemporal expression of

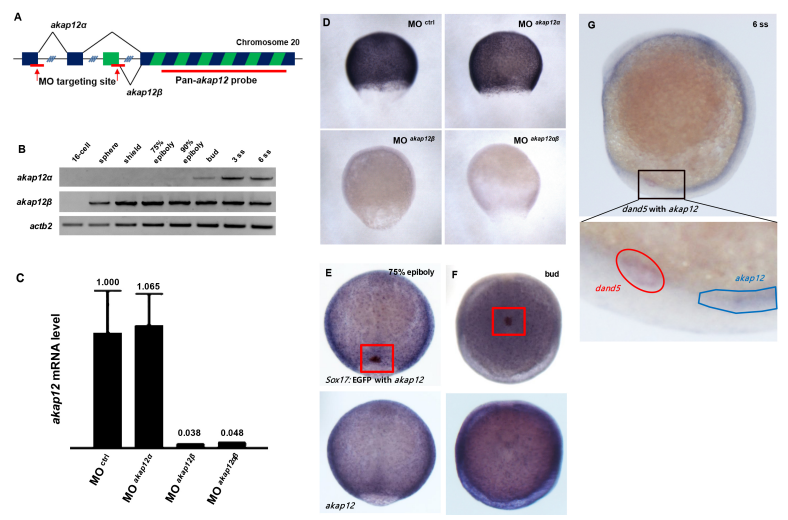

Fig. 1. KV lineage cells express akap12 $\beta$ in zebrafish. (A) The genomic locus of akap12 in zebrafish. (B) RT-PCR analysis of two akap12 isoforms. (C) qRT-PCR analysis of pan-akap12 mRNA at $75 \%$ epiboly stage in control, akap $12 \alpha$, akap $12 \beta$, and akap $12 \alpha \beta$ double morphants. (D) ISH images of pan-akap12 at $75 \%$ epiboly stage in control, akap $12 \alpha$, akap $12 \beta$, and akap $12 \alpha$ and $\beta$-double morphants. (E, F) ISH for pan-akap12 (blue) within immunostained sox 17:EGFP-positive DFCs (brown) at 75\% epiboly (E) or bud stage (F) was marked by a red rectangle. (G) Two color ISH for pan-akap12 and dand5 at 6 ss. akap12 by in situ hybridization (ISH) (Fig. 1E-G). Mesodermal cells highly expressed akap 12 mRNA as previously reported (11). Interestingly, akap12 was expressed in sox17-positive DFCs, known as the progenitor cells of KV. The observed akap12 was suggested to be akap12 $\beta$, as sox 17 -positive DFCs, which originate from non-involuting endocytic marginal cells, are present at the $75 \%$ epiboly stage (Fig. $1 \mathrm{E}, \mathrm{F}$ ). Moreover, two color ISH for akap12 and dand5, the marker of KV, revealed that akap12 expression in $\mathrm{KV}$ was not detected at the 6 somite stage (ss) when KV formation by DFC collective migration is completed (Fig. 1G). Taken together, akap12 $\beta$, but not akap $12 \alpha$, was transiently expressed in KV ascendant cells when the cluster of DFCs underwent collective migration.

\section{Heart laterality is disrupted in akap12 $\beta$ morphants}

Specific expression of akap $12 \beta$ in KV lineage cells motivated us to investigate whether akap $12 \beta$ regulates the left-sided heart orientation via fine-tuning the KV formation in zebrafish. First, the positioning of the heart was investigated by ISH for $\mathrm{cm} / \mathrm{c} 1$ in akap12 $\beta$ morphants. Akap12 $\beta$ morphants exhibited mesocardia $(\sim 20 \%)$ and dextrocardia $(\sim 12 \%)$, while control morphants showed normal heart laterality (Fig. 2A, B). Then, we evaluated KV formation in akap $12 \beta$ morphants by ISH for
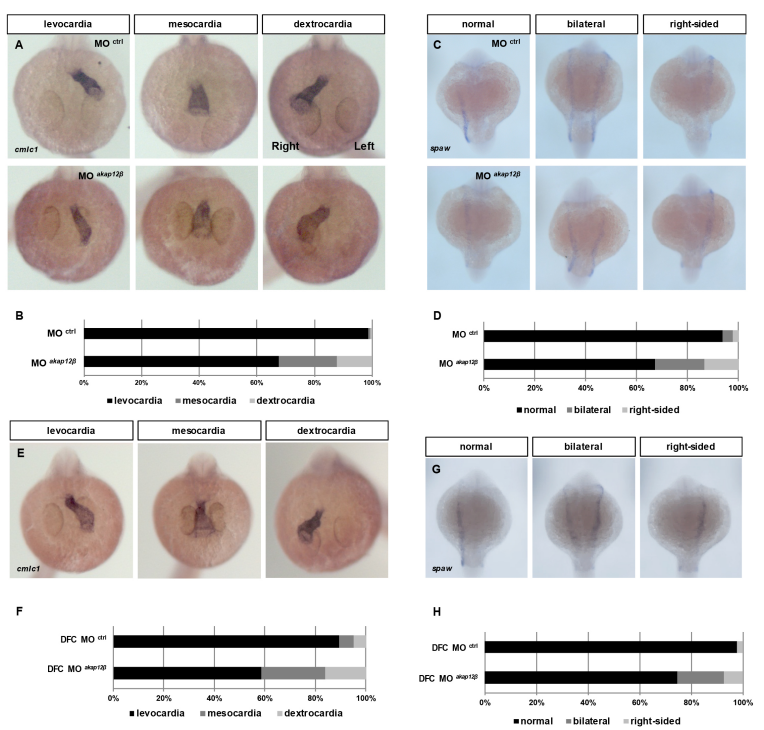

Fig. 2. Knockdown of akap $12 \beta$ disrupts organ laterality. (A) Visualization of the heart by ISH for $\mathrm{Cm} / \mathrm{C} 1$ in $30 \mathrm{hpf}$ embryos of control and akap12 $\beta$ morphants. (B) Stacked bar graph (control morphants; $n=154$, akap $12 \beta$ morphants; $n=143$ ). (C) Representative images of spaw by ISH in 18 ss embryos of control and akap $12 \beta$ morphants. (D) Stacked bar graph (control morphants; $n=174$, akap $12 \beta$ morphants; $n=195$ ). (E) Visualization of the heart by ISH for $\mathrm{cm} / \mathrm{c} 1$ in $30 \mathrm{hpf}$ embryos of DFC-specific morphants. (F) Stacked bar graph (DFC control morphants; $n=85$, DFC akap $12 \beta$ morphants; $n=94$ ). (G) Representative images of spaw by ISH for $\mathrm{cm} / \mathrm{C} 1$ in $30 \mathrm{hpf}$ embryos of DFC-specific morphants. (H) Stacked bar graph (DFC control morphants; $n=45$, DFC akap $12 \beta$ morphants; $n=67$ ). 
spaw, the nodal-related gene and a novel marker for $\mathrm{KV}$, as the abnormal KV development is a frequent cause of disorienting heart laterality. Bilateral (19\%) and right-sided (13\%) spaw expression were observed in akap $12 \beta$ morphants while aberrant spaw expression was observed in only $6 \%$ of the control morphants (Fig. 2C, D).

To investigate whether the specific downregulation of akap $12 \beta$ in the KV lineage cells such as DFCs also disrupts heart laterality, the akap $12 \beta \mathrm{MO}$ was injected into the yolk at the 128 to 512 -cell stage (DFC MO) for exclusive reduction of akap $12 \beta$ in the KV lineage cells including DFCs (16). DFCspecific injected $\mathrm{MO}$ is restricted in the boundary between the blastomeres and yolk where the KV lineage cells exist since the marginal blastomeres are connected to the yolk cell by a cytoplasmic bridge (Supplementary Fig. S1). Disrupted heart laterality $(\sim 41 \%)$ and aberrant spaw expression $(\sim 25 \%)$ were also observed in DFC-specific akap $12 \beta$ morphants, whereas low rate defects were identified in DFC control morphants (Fig. 2E-H).

\section{Akap12 $\beta$ regulates collective migration of DFCs}

Next, we validated the notochordal expression of lefty 1 , which
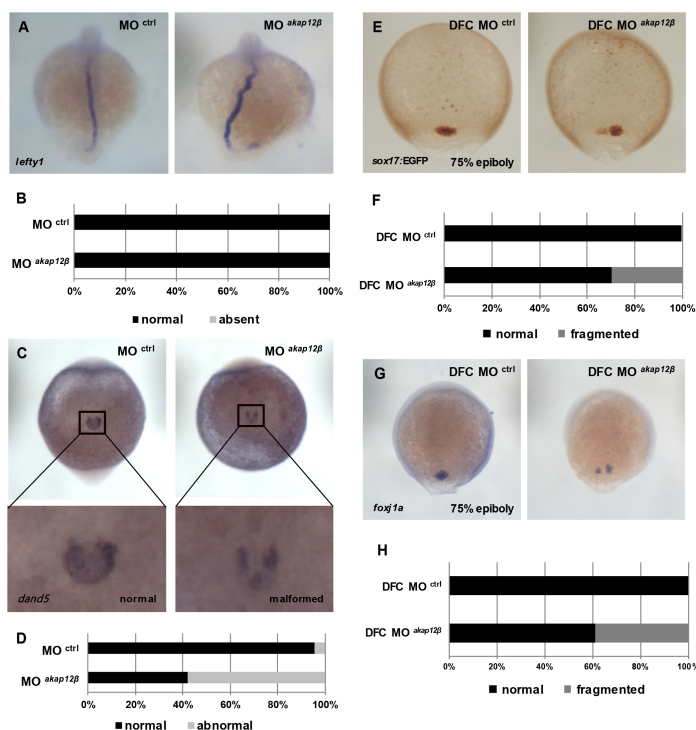

H

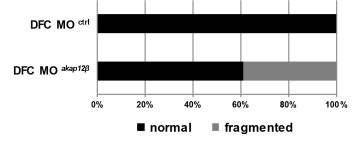

Fig. 3. Failure of collective migration of DFCs in akap $12 \beta$ morphants. (A) Visualization of lefty 1 by ISH in 18 ss embryos of control and akap $12 \beta$ morphants. (B) Stacked bar graph (control morphants; $n=$ 74, akap12 $\beta$ morphants; $n=92$ ). (C) Representative images of dand5 by ISH in 6 ss embryos of control and akap $12 \beta$ morphants. (D) Stacked bar graph (control morphants; $n=86$, akap $12 \beta$ morphants; $n=110$ ). (E) Visualization of DFC clusters by immunostaining of sox 17:EGFP in 75\% epiboly embryos of DFC-specific morphants. (F) Stacked bar graph (DFC control morphants; $n=82$, DFC akap12 $\beta$ morphants; $n=77$ ). (G) Representative images of DFC clusters by ISH for foxi1a in 75\% epiboly embryos of DFC-specific morphants. (H) Stacked bar graph (DFC control morphants; $n=22$, DFC akap12 $\beta$ morphants; $n=36$ ). functions as the midline molecular barrier to restrict nodal activity to the left lateral plate mesoderm (17). However, normal expression of lefty 1 was observed in the notochord of both control and akap12 $\beta$ morphants, regardless of dorsal curvature (Fig. 3A, B). Then, we examined dand5 expression, which is a molecular barrier of spaw by surrounding KV. The control morphants showed mostly normal dand5 expression with a horseshoe shape, however, discontinuous dand5 expression was observed in $\sim 58 \%$ of akap $12 \beta$ morphants (Fig. 3C, D). Moreover, a sox17:EGFP-positive DFC cluster, which gives rise to the $\mathrm{KV}$, was identified as fragmentation of DFC clusters in $30 \%$ of DFC akap $12 \beta$ morphants, while minimal DFC fragmentation was observed in only $\sim 1 \%$ of DFC control morphants (Fig. 3E, F). Then, we confirmed the DFC fragmentation in DFC akap12 $\beta$ morphants by ISH for foxj 1 a at $75 \%$ epiboly stage. Fragmentation of foxj1a-positive DFC clusters was observed in $\sim 39 \%$ of DFC akap $12 \beta$ morphants, while a single non-fragmented DFC cluster was identified in DFC control morphants (Fig. 3G, H).

Taken together, these data suggest that reduced akap $12 \beta$ expression in DFCs results in the disruption of asymmetric signals and heart laterality due to failure of DFC collective

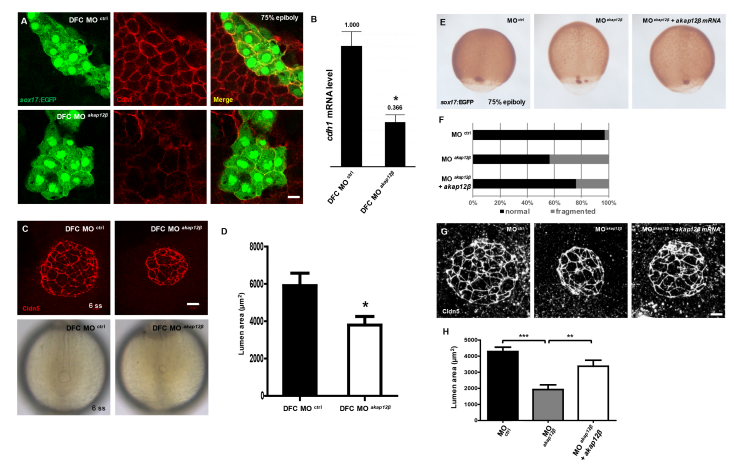

Fig. 4. Malformation of KV integrity in akap $12 \beta$ morphants and restoration of defective phenotypes in akap12 $\beta$ morphants by exogenous akap12 $\beta$ mRNA. (A) Immunostaining of Cdh1 and sox 17:EGFP in $75 \%$ epiboly embryos of DFC control and akap $12 \beta$ morphants. Scale bar, $10 \mu \mathrm{m}$. (B) qRT-PCR analysis of cdh1 mRNA in $75 \%$ epiboly embryos of DFC control and akap $12 \beta$ morphants. (C) Representative images of Cldn $5 \alpha$-immunostained KV lumen (upper) and whole embryo (lower) in 6 ss embryos of DFC control and akap $12 \beta$ morphants. Scale bar, $20 \mu \mathrm{m}$. (D) The lumen area surrounded by Cldn5 $\alpha$ is shown as the means \pm SD; ${ }^{* P}<0.05$, (DFC control morphants; $n=10$, DFC akap $12 \beta$ morphants; $n=13$ ). (E) Visualization of DFC clusters by immunostaining of sox 17:EGFP in $75 \%$ epiboly embryos of control, akap $12 \beta$, and akap $12 \beta$ mRNA-injected akap12 $\beta$ morphants. (F) Stacked bar graph (control morphants; $n=32$, akap $12 \beta$ morphants; $n=30$, akap $12 \beta$ mRNA-injected akap12 $\beta$ morphants; $n=35$ ). (G) Visualization of KV lumen by immunostaining for Cldn $5 \alpha$ in 6 ss embryos of control, akap $12 \beta$, and akap $12 \beta$ mRNA-injected akap $12 \beta$ morphants. Scale bar, $20 \mu \mathrm{m}$. (H) The lumen area surrounded by Cldn $5 \alpha$ is shown as the means $\pm \mathrm{SD} ; * * * \mathrm{P}<0.001, * * \mathrm{P}<0.01$, (control morphants; $n=10$, akap12 $\beta$ morphants; $n=10$, akap12 $\beta$ mRNA-injected akap12 $\beta$ morphants; $n=11$ ). 
migration.

\section{Reduction of cdh1 by akap12b knockdown disrupts DFC cluster integrity}

Fragmented DFC clusters are the symbolic phenotype of disrupted cell collectivity between DFCs which is maintained by Cdh1-based adherence junction (18). Thus, we evaluated Cdh1 expression in akap12 $\beta$ morphants by immunostaining at $75 \%$ epiboly stage. DFC control morphants showed high Cdh1 expression at intercellular surfaces between the DFCs. However, DFC akap $12 \beta$ morphants displayed significantly reduced Cdh1 expression within DFCs (Fig. 4A). Furthermore, not only protein expression, but also mRNA expression was decreased in akap $12 \beta$ morphants (Fig. 4B). These data suggest that the reduced Cdh1 expression within DFCs by akap12 $\beta$ downregulation could lead to KV malformation due to DFC fragmentation. Then, we examined how DFC fragmentation affects KV formation in akap $12 \beta$ morphants. Interestingly, we identified that the size of KV in DFC akap12 $\beta$ morphants was relatively smaller than that of DFC control morphants using differential interference contrast images of living embryos. Consistent with these observations, KV apical lumen area encompassed by Cldn5 $\alpha$ was significantly reduced in DFC akap12 $\beta$ morphants (0.63-fold vs. DFC control morphants, Fig. 4C, D).

Finally, akap $12 \beta$ mRNA was injected with MOs to rescue the phenotypes of akap $12 \beta$ morphants. Fragmentation of DFC clusters was restored by exogenous akap $12 \beta$. Akap $12 \beta$ mRNA injected akap $12 \beta$ morphants showed a higher rate of normally formed single non-fragmented DFC cluster $(\sim 76 \%)$ than akap $12 \beta$ morphants ( $\sim 7 \%$ ) (Fig. 4E, F). Consistently, Exogenous akap $12 \beta$ mRNA also increased the KV lumen area in akap $12 \beta$ morphants (1.75-fold vs. akap12 $\beta$ morphants, Fig. 4G, H). Taken together, these data suggest that the loss of cell collectivity within DFCs reduces KV size and this reduction finally results in heart laterality defects in akap $12 \beta$ morphants.

\section{DISCUSSION}

The current study investigated the role of akap $12 \beta$ and akap $12 \alpha$ in heart laterality establishment, providing the first experimental evidence that akap12 $\beta$, not akap $12 \alpha$, might play a role in human heart heterotaxy. We showed that akap $12 \beta$ is the major isoform of akap12 during embryogenesis and is expressed in DFCs, ascendant cells of KV. Knockdown of akap $12 \beta$ led to the reduced $c d h 1$ expression in DFCs which resulted in loss of cell collectivity within DFCs. Finally, fragmented DFC clusters gave rise to smaller KV and malformed KV failed to establish proper heart laterality.

AKAP12 was first identified as an autoantigen in myasthenia gravis, so it was named Gravin (7). In the present study, we investigated the specific role of akap $12 \beta$ in heart laterality regulation. In akap12 $\alpha$ morphants, a single sox 17:EGFPpositive DFC cluster was preserved and reduction of $c d h 1$ was not observed during KV development (Supplementary Fig. S2 and S3A, B). In this regard, Gelman et al. examined the expression of AKAP12 isoforms in the internal organs including the heart of human and mouse and showed by immunostaining that Akap12 is expressed in the heart during fetal stage (19). Besides, Streb et al. reported that Akap12 $\alpha$ and Akap $12 \beta$ are differentially regulated by independent promoters in different tissues and cells (14). In the recent study, two kinds of de novo loss of function mutations of AKAP12 within the exon shared by both isoforms were identified as high-risk mutations in CHD patients of left ventricular obstruction and heterotaxy such as dextrocardia, respectively (15). Interestingly, sudden death was observed in $3 \%$ to $4 \%$ of 4 -month-old Akap12-null mice which carry the deletion of the common exon of Akap12 isoforms and cardiomegaly was commonly identified in all those cases by autopsy (20). Accordingly, we hypothesized that AKAP12 $\alpha$ might control the pure heart development considering it as the major isoform of $A K A P 12$ in the heart (14) and $A K A P 12 \beta$ might regulate proper heart positioning depending upon our current investigation, respectively. Further investigation is necessary to define the exact role of AKAP12 isoforms in heart development.

Our group reported that AKAP12 regulates junctional protein expression such as E-Cadherin, VE-Cadherin, Claudin-1, Occludin, and ZO-1 in diverse systems (8-10, 12). In zebrafish, Cdh1-mediated cell adhesion between adjacent DFCs is essential for their collective migration followed by KV morphogenesis $(2,18)$. In this regard, we also observed reduction in cdh1 mRNA and protein expression in akap12 $\beta$-deficient zebrafish. Moreover, exogenous akap12 $\beta$ mRNA not only maintained a single non-fragmented DFC cluster and the size of $\mathrm{KV}$ but also restored cdh1 mRNA expression in akap12 $\beta$ morphants (Fig. S3C). However, we focused on the role of akap $12 \beta$ in the establishment of heart laterality in the current study so further investigations including mechanism(s) of independent expression of akap12 isoforms and regulation of $c d h 1$ expression by akap $12 \beta$ should be necessary.

Recent studies have revealed that asymmetric distribution of hypoxia contributes to dorsoventral axis establishment during embryogenesis of sea urchin and that retinoic acid (RA) is involved (21-23). Moreover, our group previously reported that partial oxygen pressure regulates AKAP12 expression and that RA induces AKAP12 expression in CNS injury repair (9, 24) and we hypothesized that gradation of such factors could regulate the spatiotemporal expression of akap12 isoforms. Relating to cdh1 expression, our group reported that AKAP12 induced by reoxygenation and/or RA suppresses SNAI1, a master transcription factor for epithelial-mesenchymal transition, via the non-Smad pathway during the recovery of CNS injury and that AKAP12 knockdown increases SNAI1 expression in ARPE-19 epithelial cell line (24). Considering Snai1 as a well-known transcriptional repressor for E-cadherin expression (25) and the possible role(s) of Snai1 in asymmetric 
development $(26,27)$, we suggest that reduced Cdh1 expression in akap12 $\beta$ morphants might be mediated by enhanced Snai1 expression.

In addition to DFC collective migration, DFC numbers and ciliogenesis in KV are crucial for heart laterality (17). Our data indicated that proliferation of DFCs in akap $12 \beta$ morphants was comparable with control morphants and the number of cilia in akap $12 \beta$ morphants was similar to that in control morphants considering the size of the KV lumen (Supplementary Fig. S4, 5). Malformed KV affected not only heart laterality but also other asymmetric internal organs since we also observed aberrant pancreas positioning in akap12 $\beta$ morphants (Supplementary Fig. S6). These data, together with other current data, suggest that loss of function mutation of AKAP12 might be linked to diverse heterotaxy.

Given genetic evidences of AKAP12 loss of function mutation in certain $\mathrm{CHD}$ patients, our data indicating that akap $12 \beta$, not akap $12 \alpha$, specifically regulates heart laterality via regulation of $c d h 1$ expression in DFCs in zebrafish could be extended to the regulation of heart laterality and asymmetric development of internal organs in humans.

\section{MATERIALS AND METHODS}

\section{Zebrafish}

Tuebingen wild-type zebrafish and transgenic sox17:egfp ${ }^{5870}$ zebrafish (Tg(sox17:egfp)) were previously described (16). All zebrafish work was carried out in accordance with protocols approved by the Institutional Animal Care and Use Committees of Seoul National University.

\section{Morpholino injection}

The protocol of $\mathrm{MO}$ injection into zebrafish embryos was previously described (13). Briefly, splice-blocking MOs were injected into the yolk at one-cell stage for whole embryo knockdown or at 128-512-cell stage for DFC-specific knockdown as indicated. Translation-blocking MOs were used to rule out the off-target effects (Supplementary Fig. S7). MOs for akap12 $\alpha$ and akap $12 \beta$ were previously described (13).

\section{In vitro transcription}

PCR-amplified akap12 $\beta$ was cloned into pCS2 + vector (28). $5^{\prime}$-capped and poly(A)-tailed mRNAs were generated using mMessage mMachine ultra kit (Ambion). 80-120 pg of akap12 $\beta$ mRNA was co-injected with akap $12 \beta \mathrm{MO}$. Sequences of primers for akap $12 \beta$ cloning are summarized in Supplementary Table S1.

\section{RNA isolation and quantitative RT-PCR}

The protocols of qRT-PCR and RT-PCR were previously described (29). Total RNA was isolated from zebrafish embryos at indicated stages with TRIzol reagent (Invitrogen) and cDNA was obtained from $2 \mu \mathrm{g}$ of total RNA using MMLV reverse transcriptase (Promega). qRT-PCR was then performed using StepOnePlus RT-PCR system (Applied Biosystems) with RealHelix qPCR kit (NanoHelix). Relative mRNA expression levels were calculated by the comparative $2^{-\Delta \Delta C t}$ method. Actb2 and eef1a1/1 served as internal controls. Primer sequences for qRT-PCR are summarized in Supplementary Table S1.

\section{Whole-mount ISH and immunostaining}

The protocol of ISH was previously described (16). Specific regions of lefty 1 and foxj1a were cloned into pGEM T easy vector (Promega). ISH probe vectors for $\mathrm{cm} / \mathrm{c} 1$, dand5, spaw, and pan-akap12 were previously described $(12,16)$ and primer sequences of lefty 1 and foxj1a for ISH probe vectors are summarized in Supplementary Table S1. The protocol of whole-mount immunostaining for $\operatorname{Tg}(\operatorname{sox} 17$ :egfp) embryos was described previously (16). Mouse anti-Cdh1 (1:200, BD Biosciences) and goat anti-mouse AF546 (1:1000, Invitrogen) were used for immunofluorescence. Stained embryos were mounted in glycerol and images were obtained by an AxioCam ICC-1 camera (Zeiss) on a Stemi 2000C (Zeiss) for immunohistochemistry and an LSM700 confocal microscope (Zeiss) for immunofluorescence, respectively, and processed using ZEN 2012 software (Zeiss).

\section{Statistical analysis}

Measurement of KV lumen area was described previously (16). The data are presented as means \pm SD and analyzed with Prism 5 (GraphPad Software, Inc.). The data in Fig. 4B and 4D were analyzed by two-tailed Student's t-test and the data in Fig. $4 \mathrm{H}$ were analyzed by one-way ANOVA test.

\section{ACKNOWLEDGEMENTS}

The authors appreciated Emeritus Prof. Kyu-Won Kim (Seoul National University, Seoul, Korea) for mentoring and providing expertise on this study. J.-g.K. designed the research, performed experiments and cared for zebrafish; H.-H.K. analyzed data and helped write the manuscript; S.J.B. wrote the manuscript and supervised the research. This work was supported by Basic Science Research Program (NRF-2017R1A6A3A11032239) through the NRF funded by the Korean Ministry of Education and the Medical Research Center Program (2014R1A5A20 009936) through the NRF funded by the Korean Ministry of Science, ICT and Future Planning (MSIP).

\section{CONFLICTS OF INTEREST}

The authors have no conflicting interests.

\section{REFERENCES}

1. Essner JJ, Amack JD, Nyholm MK, Harris EB and Yost HJ (2005) Kupffer's vesicle is a ciliated organ of asymmetry in the zebrafish embryo that initiates left-right development 
of the brain, heart and gut. Development 132, 1247-1260

2. Matsui T, Thitamadee S, Murata T et al (2011) Canopy1, a positive feedback regulator of FGF signaling, controls progenitor cell clustering during Kupffer's vesicle organogenesis. Proc Natl Acad Sci U S A 108, 9881-9886

3. Oteiza $P$, Koppen $M$, Concha $M L$ and Heisenberg $C P$ (2008) Origin and shaping of the laterality organ in zebrafish. Development 135, 2807-2813

4. Desgrange A, Le Garrec JF and Meilhac SM (2018) Left-right asymmetry in heart development and disease: forming the right loop. Development 145, dev162776

5. van der Linde D, Konings EE, Slager MA et al (2011) Birth prevalence of congenital heart disease worldwide: a systematic review and meta-analysis. J Am Coll Cardiol 58, 2241-2247

6. Hartill VL, van de Hoek G, Patel MP et al (2018) DNAAF1 links heart laterality with the AAA + ATPase RUVBL1 and ciliary intraflagellar transport. Hum Mol Genet 27, 529-545

7. Gelman IH (2012) Suppression of tumor and metastasis progression through the scaffolding functions of SSeCKS/ Gravin/AKAP12. Cancer Metastasis Rev 31, 493-500

8. Choi YK, Kim JH, Kim WJ et al (2007) AKAP12 regulates human blood-retinal barrier formation by downregulation of hypoxia-inducible factor-1alpha. J Neurosci 27, 44724481

9. Lee S-W, Kim WJ, Choi YK et al (2003) SSeCKS regulates angiogenesis and tight junction formation in blood-brain barrier. Nat Med 9, 900

10. Cha JH, Wee HJ, Seo JH et al (2014) AKAP12 mediates barrier functions of fibrotic scars during CNS repair. PLoS One 9, e94695

11. Weiser DC, Pyati UJ and Kimelman D (2007) Gravin regulates mesodermal cell behavior changes required for axis elongation during zebrafish gastrulation. Genes Dev $21,1559-1571$

12. Kwon HB, Choi YK, Lim JJ et al (2012) AKAP12 regulates vascular integrity in zebrafish. Exp Mol Med 44, 225-235

13. Kim HH, Kim JG, Jeong J, Han SY and Kim KW (2014) Akap12 is essential for the morphogenesis of muscles involved in zebrafish locomotion. Differentiation 88, 106-116

14. Streb JW, Kitchen CM, Gelman IH and Miano JM (2004) Multiple promoters direct expression of three AKAP12 isoforms with distinct subcellular and tissue distribution profiles. J Biol Chem 279, 56014-56023

15. Jin SC, Homsy J, Zaidi S et al (2017) Contribution of rare inherited and de novo variants in 2,871 congenital heart disease probands. Nat Genet 49, 1593-1601

16. Kim JG, Bae SJ, Lee HS, Park JH and Kim KW (2017)
Claudin5a is required for proper inflation of Kupffer's vesicle lumen and organ laterality. PLoS One 12, e0182047

17. Joseph Yost H (1999) Diverse initiation in a conserved left-right pathway? Curr Opin Genet Dev 9, 422-426

18. Tay HG, Schulze SK, Compagnon J et al (2013) Lethal giant larvae 2 regulates development of the ciliated organ Kupffer's vesicle. Development 140, 1550-1559

19. Gelman IH, Tombler E and Vargas J (2000) A Role for SSeCKS, a major protein kinase C substrate with tumour suppressor activity, in cytoskeletal architecture, formation of migratory processes, and cell migration during embryogenesis. Histochem J 32, 13-26

20. Akakura S, Huang C, Nelson PJ, Foster B and Gelman IH (2008) Loss of the SSeCKS/Gravin/AKAP12 gene results in prostatic hyperplasia. Cancer Res 68, 5096-5103

21. Sugrue KF, Sarkar AA, Leatherbury L and Zohn IE (2019) The ubiquitin ligase HECTD1 promotes retinoic acid signaling required for development of the aortic arch. Dis Model Mech 12, dmm036491

22. Vroomans RMA and Ten Tusscher K (2017) Modelling asymmetric somitogenesis: Deciphering the mechanisms behind species differences. Dev Biol 427, 21-34

23. Robichaux JP, Fuseler JW, Patel SS, Kubalak SW, Hartstone-Rose A and Ramsdell AF (2016) Left-right analysis of mammary gland development in retinoid $X$ receptor-alpha+/- mice. Philos Trans R Soc Lond B Biol Sci 371, 20150416

24. Cha JH, Wee HJ, Seo JH et al (2014) Prompt meningeal reconstruction mediated by oxygen-sensitive AKAP12 scaffolding protein after central nervous system injury. Nat Commun 5, 4952

25. Cano A, Pérez-Moreno MA, Rodrigo I et al (2000) The transcription factor snail controls epithelial-mesenchymal transitions by repressing E-cadherin expression. Nat Cell Biol 2, 76-83

26. Gupta K, Pilli VS and Aradhyam GK (2016) Left-right axis asymmetry determining human cryptic gene is transcriptionally repressed by snail. BMC Dev Biol 16, 39

27. Collins MM, Baumholtz Al, Simard A, Gregory M, Cyr DG and Ryan AK (2015) Claudin-10 is required for relay of left-right patterning cues from Hensen's node to the lateral plate mesoderm. Dev Biol 401, 236-248

28. Bae SJ, Shin MW, Kim RH et al (2017) Ninjurin1 assembles into a homomeric protein complex maintained by N-linked glycosylation. J Cell Biochem 118, 2219-2230

29. Bae SJ, Shin MW, Son T et al (2019) Ninjurin1 positively regulates osteoclast development by enhancing the survival of prefusion osteoclasts. Exp Mol Med 51, 7 